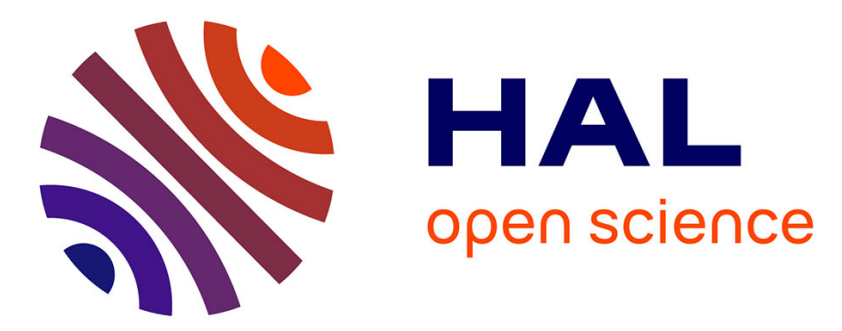

\title{
Étude de la préparation et des propriétés électriques de couches minces semiconductrices d'antimoniure d'aluminium
}

\author{
J.P. David, L. Capella, L. Laude, S. Martinuzzi
}

\section{- To cite this version:}

J.P. David, L. Capella, L. Laude, S. Martinuzzi. Étude de la préparation et des propriétés électriques de couches minces semiconductrices d'antimoniure d'aluminium. Revue de Physique Appliquée, 1966, 1 (3), pp.172-178. 10.1051/rphysap:0196600103017201 . jpa-00242710

HAL Id: jpa-00242710

https://hal.science/jpa-00242710

Submitted on 1 Jan 1966

HAL is a multi-disciplinary open access archive for the deposit and dissemination of scientific research documents, whether they are published or not. The documents may come from teaching and research institutions in France or abroad, or from public or private research centers.
L'archive ouverte pluridisciplinaire HAL, est destinée au dépôt et à la diffusion de documents scientifiques de niveau recherche, publiés ou non, émanant des établissements d'enseignement et de recherche français ou étrangers, des laboratoires publics ou privés. 


\title{
ÉTUDE DE LA PRÉPARATION ET DES PROPRIÉTÉS ÉLECTRIQUES DE COUCHES MINCES SEMICONDUCTRICES D'ANTIMONIURE D'ALUMINIUM
}

\author{
Par J. P. DAVID, L. CAPELLA, L. LAUDE et S. MARTINUZZI, \\ Laboratoire de Physique S.P.C.N. III, Laboratoire d'Héliotechnique, Laboratoire de Cristallographie Physique, \\ Faculté des Sciences de Marseille.
}

\begin{abstract}
Résumé. - Nous avons réalisé des couches minces du composé AlSb présentant des propriétés semiconductrices semblables à celles du matériau massif. La technique de préparation utilise l'évaporation simultanée des éléments constituants dans des proportions stoechiométriques. Pour des températures de recuit supérieures ou égales à $650^{\circ} \mathrm{C}$, seul le composé AlSb cristallise à partir des phases liquides des composants, alors qu'il est accompagné d'une phase antimoine résiduelle pour des recuits effectués entre 520 et $650^{\circ} \mathrm{C}$.

Les couches sont toutes de type $p$ et leur conductibilité varie entre 0,05 et $0,5 \Omega^{-1} \mathrm{~cm}^{-1}$ à $250^{\circ} \mathrm{C}$. L'étude de la transmission (I. R. et visible) et de la réflexion optique de ces couches conduit à une largeur de bande interdite optique de $1,90 \mathrm{eV}$ associée à une transition indirecte nécessitant une énergie d'activation de 1,50 eV. Ces résultats sont confirmés par la variation spectrale de la photoconductibilité.
\end{abstract}

Abstract. - AlSb thin films, showing semiconducting properties similar to those of massive material, have been obtained. The preparation uses a coevaporation process of stoichiometric amounts of components. For temperatures higher than $650^{\circ} \mathrm{C}$, only the compound $\mathrm{AlSb}$ is crystallized through liquid phases of components, while a residual antimony phase coexists with it for annealing between 520 and $650^{\circ} \mathrm{C}$.

All the films are $p$ type and their conductivities range from 0.05 to $0.5 \Omega^{-1} \mathrm{~cm}^{-1}$ at $20^{\circ} \mathrm{C}$. I. R. and visible transmission and reflectivity were studied; this permitted the determination of the optical forbidden band gap found at $1.90 \mathrm{eV}$, associated with an indirect transition for a $1.50 \mathrm{eV}$ activation energy. Measurements of the photoconduction spectral sensitivity are in good agreement with these results.

I. Introduction. - Parallèlement au travail d'exploration que nous avons effectué sur les couches de GaAs et de Cd'Te, nous avons entrepris l'étude des possibilités des couches minces d'antimoniure d'aluminium dans la réalisation de photopiles.

Le choix de ce matériau provient essentiellement de la valeur élevée de son énergie d'activation (1,52 $\mathrm{eV}$ à température ambiante), car les mobilités dans le matériau massif sont relativement faibles. Ce composé n'a d'ailleurs pas été utilisé jusqu'à présent à cause de son caractère hygroscopique très marqué et des difficultés rencontrées au cours des dopages [1] à [20].
En couches minces, quelques études partielles ont été faites d'abord par Michel [22], Presnov et Synorov [23], Sorokin [24] puis simultanément par J. E. Johnson [25] et notre équipe de recherche [26]. La plus grande difficulté rencontrée réside dans la protection de ces couches contre l'action de la vapeur d'eau et de l'oxygène. Ce problème semble être résolu par le dépôt d'une couche de monoxyde de silicium sur la couche d'AlSb à la température ambiante. Nous nous sommes proposé dans une première phase d'explorer les procédés de préparation de ces couches ainsi que leurs propriétés électriques, optiques et photoélectriques. 
Les premiers résultats obtenus sont rassemblés dans cet exposé.

II. Préparation des couches. - a) ConsidéraTIONS GÉNÉralEs. - Les évaporations s'effectuent sous une pression de $10^{-6}$ Torr. A cause des différences très marquées entre les tensions de vapeur de l'aluminium et de l'antimoine et de l'attaque très rapide du composé par la vapeur d'eau, nous n'avons retenu que l'évaporation thermique simultanée ou décalée des éléments constituants, dans des propor-

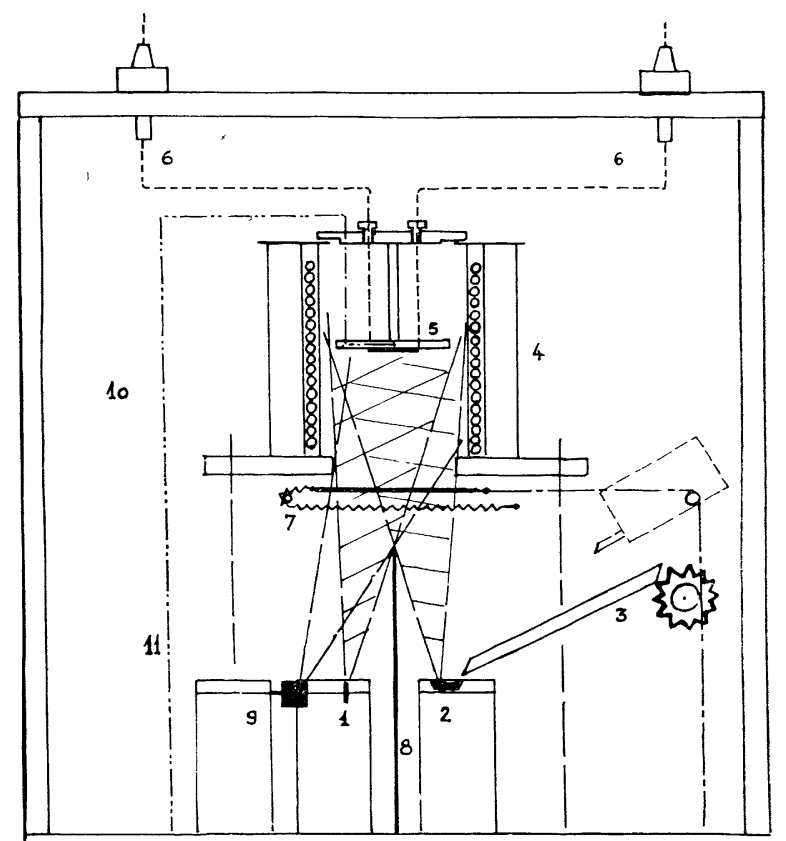

Fig. 1. tions stoechiométriques, l'antimoine étant projeté par évaporation flash.

Le montage de préparation est schématisé sur la figure 1.

Les couches sont condensées sur un support de silice placé dans un four dont la température peut atteindre $1000^{\circ} \mathrm{C}$. Des contacts électriques en aluminium ont été déposés préalablement sur ce support. Ils permettent de suivre au cours des recuits la résistance électrique.

Le choix de l'aluminium est imposé par l'agressivité dont fait preuve l'antimoniure d'aluminium à l'égard de tous les métaux utilisés habituellement, surtout au-dessus de $600^{\circ} \mathrm{C}$.

Les épaisseurs des couches condensées et recuites varient de 0,10 à $0,40 \mu$, et leur structure est étudiée aux rayons $\mathrm{X}$ et au microscope métallographique.

Nous avons été guidés dans le choix des températures de condensation $T_{\mathrm{S}}$ et de recuit $T_{\mathrm{R}}$ par le diagramme de phases établis par Guertler et Bergman [28] et par Liu et Peretti [29].

Les résultats que nous avons obtenus pour les différentes valeurs de $T_{\mathrm{R}}$ et $T_{\mathrm{S}}$ sont les suivants

b) Couches préparées par évaporation simulTANÉE d'Al ET DE Sb.

1) $T_{\mathrm{S}}$ et $T_{\mathrm{R}}<250{ }^{\circ} \mathrm{C}$. - Les diagrammes de rayons $\mathrm{X}$ indiquent une structure pratiquement amorphe et ne décèlent pas la présence du composé AlSb.

On note toutefois une faible cristallisation due à l'antimoine.

2) $T_{\mathrm{S}}<520^{\circ} \mathrm{C}$ et $520^{\circ} \mathrm{C}<T_{\mathrm{R}}<650^{\circ} \mathrm{C}$. Quelle que soit alors $T_{\mathrm{S}}$, la structure des couches sera uniquement fonction de la température de recuit $T_{\mathrm{R}}$ et de la durée des recuits, en l'absence

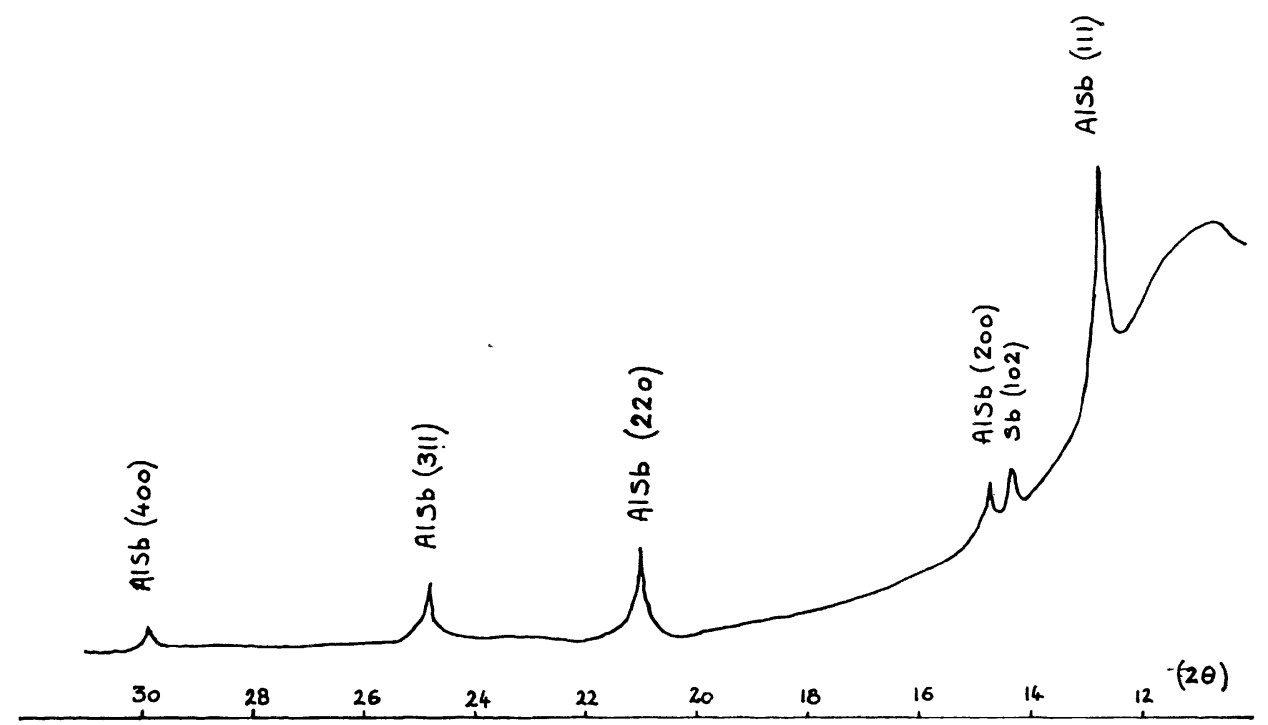

FIG. 2. 
desquels nous avons vérifié que les couches étaient toujours amorphes et non stoechiométriques.

Par contre les radiogrammes de rayons $X$ décèlent la présence d'AlSb (fig. 2) mais associé à une phase antimoine, dont l'importance décroît quand $T_{R}$ s'élève.

Il semble donc que la cristallisation du composé s'opère à partir de celle de $\mathrm{Sb}$, chaque cristallite de celui-ci étant remplacé progressivement par un cristallite d'AlSb. La nature discontinue et aléatoire de la cristallisation du composé à ces températures expliquerait donc l'augmentation très importante de la résistivité électrique et justifierait les résultats des essais suivants.

3) $520^{\circ} \mathrm{C}<T_{\mathrm{s}}<650^{\circ} \mathrm{C}$ et $T_{\mathrm{R}}<650^{\circ} \mathrm{C}$. - Dès la fin de leur préparation et avant tout recuit, les diagrammes des rayons $\mathrm{X}$ signalent la présence d'AlSb comme précédemment. Des recuits prolongés provoquent, aux environs de $650{ }^{\circ} \mathrm{C}$, une cristallisation uniforme de la phase AlSb qui se traduit par une augmentation très brutale de la résistance électrique, comme nous le verrons plus loin. Pour $T_{\mathrm{R}}>650^{\circ} \mathrm{C}$, il semble que la couche soit stabilisée puisqu'on n'observe aucune évolution irréversible de la résistance électrique en fonction de la température.

Enfin, pour $T_{\mathrm{s}}=650^{\circ} \mathrm{C}$, nous obtenons directement et sans recuit la phase AlSb.

Ces résultats sont en accord avec le diagramme des phases du composé dont la cristallisation s'opère à partir des phases liquides de $\mathrm{Al}$ et de $\mathrm{Sb}$, dont les points de fusion sont respectivement de $657^{\circ} \mathrm{C}$ et $630^{\circ} \mathrm{C}$.

c) Couches réalisées par évaporation décaLÉE. - Par ailleurs dans la technique précédente, nous avons observé au microscope métallographique un grand nombre de cristallites accolés au support par (111) sur les portions de contacts d'aluminium recouverts par la couche (fig. 3). Ceci nous a suggéré la préparation par évaporation décalée des éléments composants.

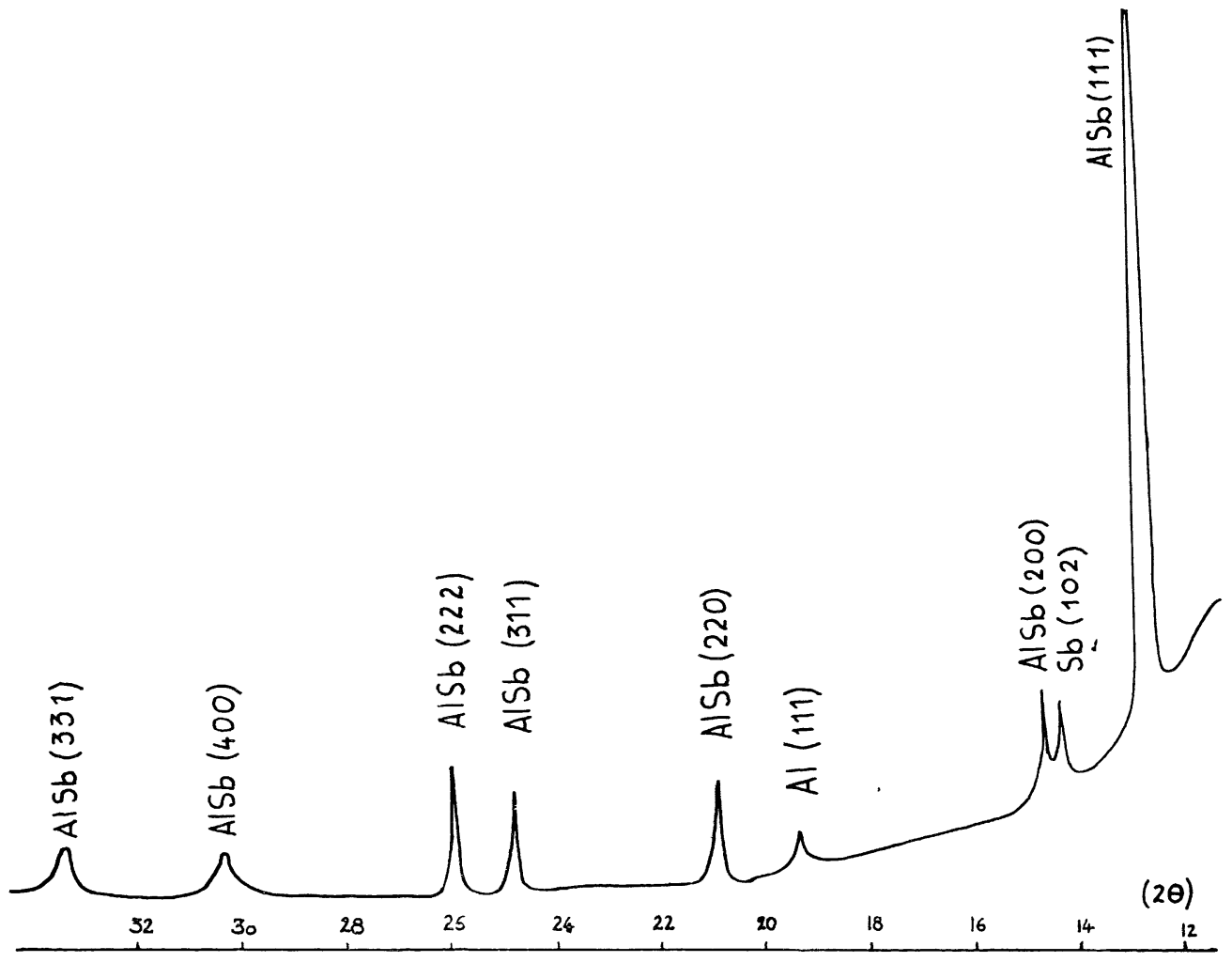

Fig. 3.

On évapore successivement :

- l'aluminium seul ;

- puis simultanément les deux éléments jusqu'à épuisement de l'aluminium ;

- enfin l'antimoine résiduel ;

(Les quantités étant toujours stoéchiométriques.)

Pour des températures de recuit inférieures à $740^{\circ} \mathrm{C}$, la diffusion des éléments $\mathrm{Al}$ et $\mathrm{Sb}$ n'est pas suffisante et nous n'obtenons pas le composé.

Par contre, pour $T_{\mathrm{R}} \geqslant 740^{\circ} \mathrm{C}$, la diffraction des rayons $\mathrm{X}$ indique la présence du composé AlSb. La conductibilité électrique, qui était métallique dans le premier cas, devient alors semiconductrice. 
d) Conclusion. - Par évaporation simultanée des éléments constituants nous avons obtenu des couches pratiquement stoéchiométriques après recuit à $650{ }^{\circ} \mathrm{C}$. Le résultat est identique avec les évaporations décalées, après recuit à $740{ }^{\circ} \mathrm{C}$, mais en plus on observe un accolement préférentiel suivant (111).

III. Propriétés électriques. - Les valeurs élevées des résistances des couches après stabilisation ont rendu très difficiles les mesures d'effet Hall. Aussi seule la conductibilité électrique a pu être étudiée en fonction de la température et surtout au cours des recuits.

Signalons tout de suite que toutes les couches obtenues sont de type $p$, quelles que soient les conditions de préparation.

a) Variation de la résistance au cours des RECUITS.

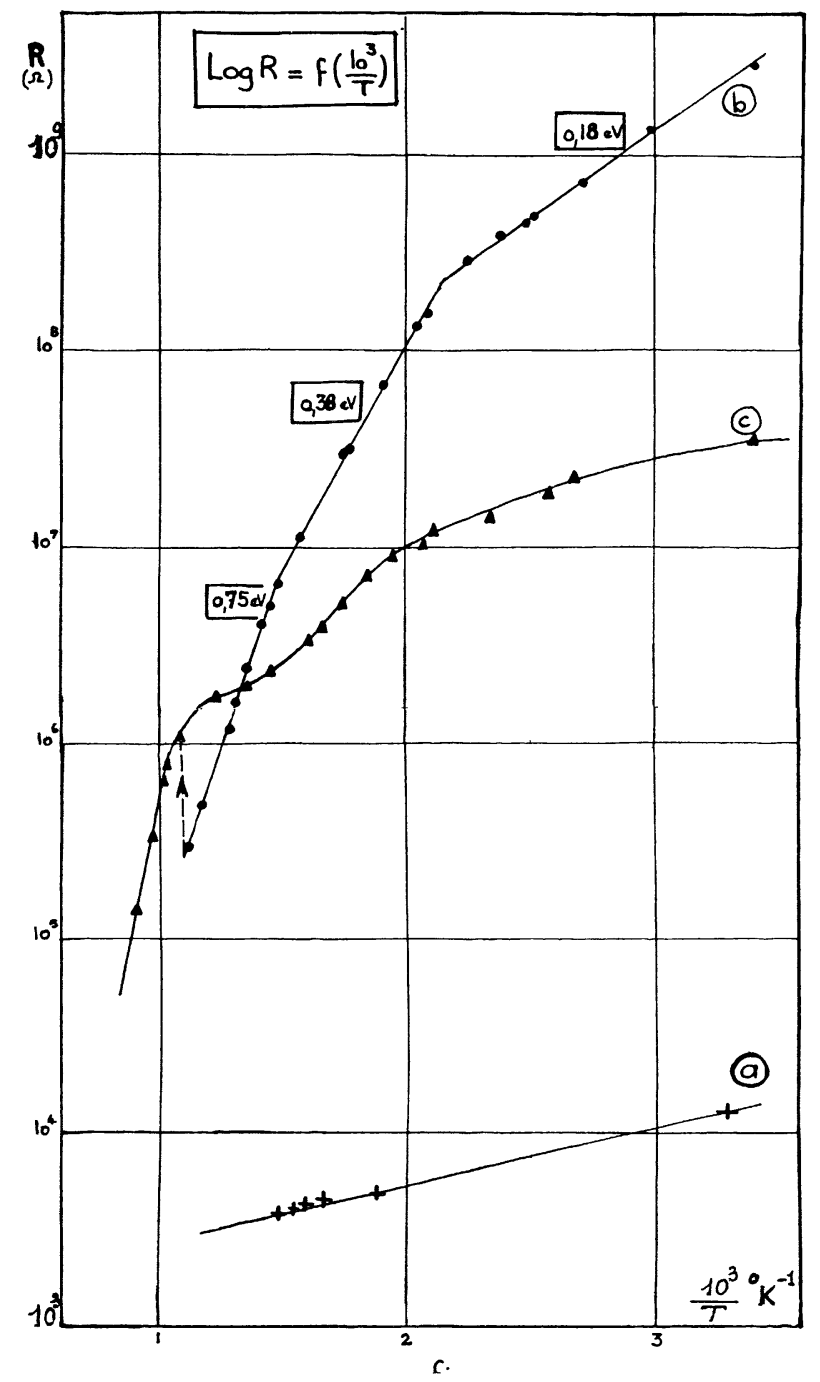

FIG. 4.
$T_{\mathrm{S}}$ et $T_{\mathrm{R}}<520^{\circ} \mathrm{C}$. - Pour ces couches les propriétés semiconductrices sont peu marquées et les résistances sont faibles $\left(10^{2} \Omega\right.$ ) (fig. $\left.4 a\right)$.

$T_{\mathrm{S}}<520^{\circ} \mathrm{C}$ et $520^{\circ} \mathrm{C}<T_{\mathrm{R}} 650^{\circ} \mathrm{C}$. - - Au cours $\mathrm{du}$ recuit la résistance électrique augmente brutalement d'un facteur $10^{4}$ environ (fig. 4b). Les courbes $\log R=f(1 / T)$ mettent en évidence trois niveaux :

$$
\begin{aligned}
& -0,18+0,02 \mathrm{eV} \\
& -0,38+0,05 \mathrm{eV} . \\
& -0,75+0,05 \mathrm{eV}
\end{aligned}
$$

Les deux premiers niveaux concordent avec les résultats donnés par Johnson [28] entre $170{ }^{\circ} \mathrm{C}$ et $500^{\circ} \mathrm{C}$, pour des couches minces d'AlSb. Signalons ici que, contrairement à cet auteur, nous avons trouvé pour des couches préparées dans les mêmes conditions de température une phase antimoine accompagnant la phase AlSb.

Le troisième niveau apparaît entre 450 et $650^{\circ} \mathrm{C}$ et semble correspondre au niveau d'impureté trouvé par Blunt et al. [4] et par Nasledov [13]. Nous n'avons pas élucidé l'origine de ces niveaux, du fait qu'ils disparaissent après recuit à plus de $650^{\circ} \mathrm{C}$.

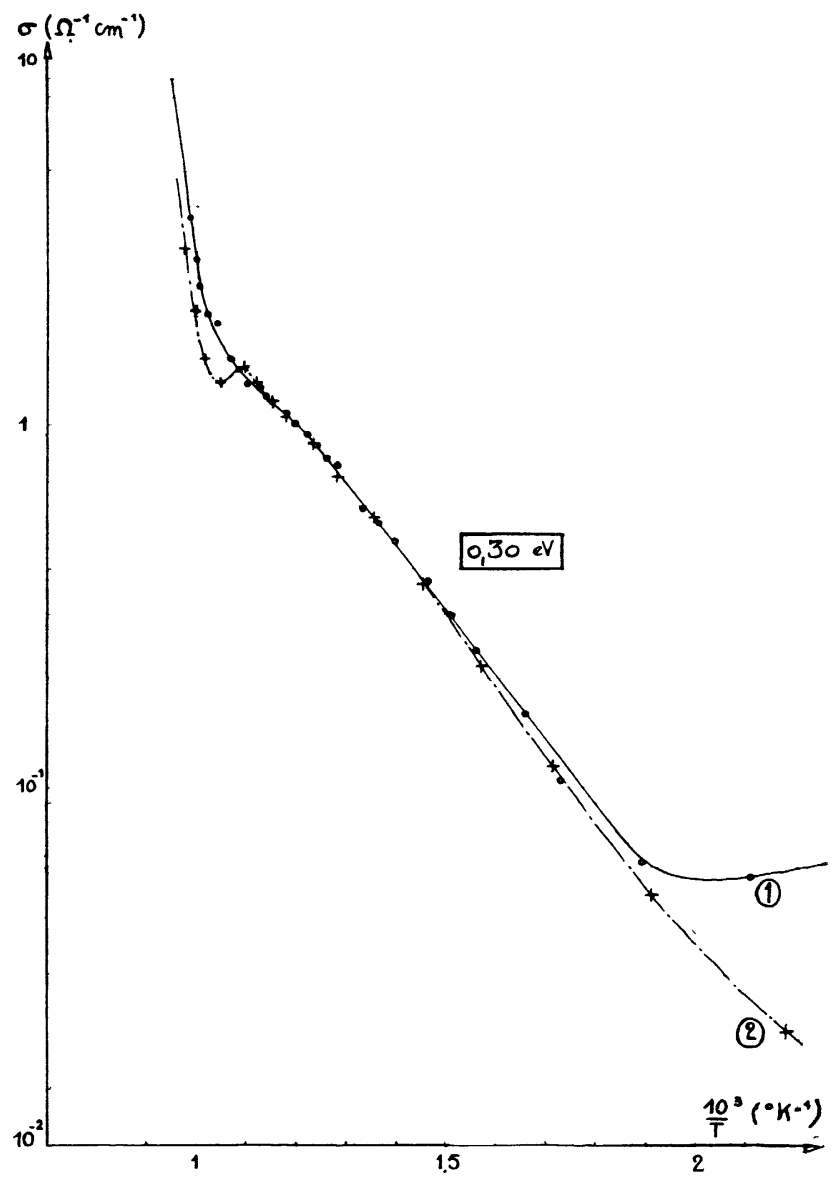

FIG. 5. 
d) $520^{\circ} \mathrm{C}<T_{\mathrm{S}}<650^{\circ} \mathrm{C}$ et $T_{\mathrm{R}}>650^{\circ} \mathrm{C}$. Lorsque $T_{R}$ atteint $650 \mathrm{o}$, la résistance de la couche est multipliée brusquement par un facteur 10 (fig. 4c).

La couche semble alors se stabiliser, les variations de $\sigma$ avec $T_{R}$ étant réversibles.
Nous avons alors étudjé l'évolution de $\sigma$ en fonction de $1 / T$, et les résultats sont schématisés par les figures 5 et 6 . Aux températures supérieures à $650^{\circ} \mathrm{C}$ la conduction est intrinsèque ; entre $650^{\circ} \mathrm{C}$ et $250^{\circ} \mathrm{C}$ apparaît un niveau donneur à $0,30 \mathrm{eV}$. Après la zone de conduction mixte $\left(250^{\circ} \mathrm{C}-20^{\circ} \mathrm{C}\right)$,

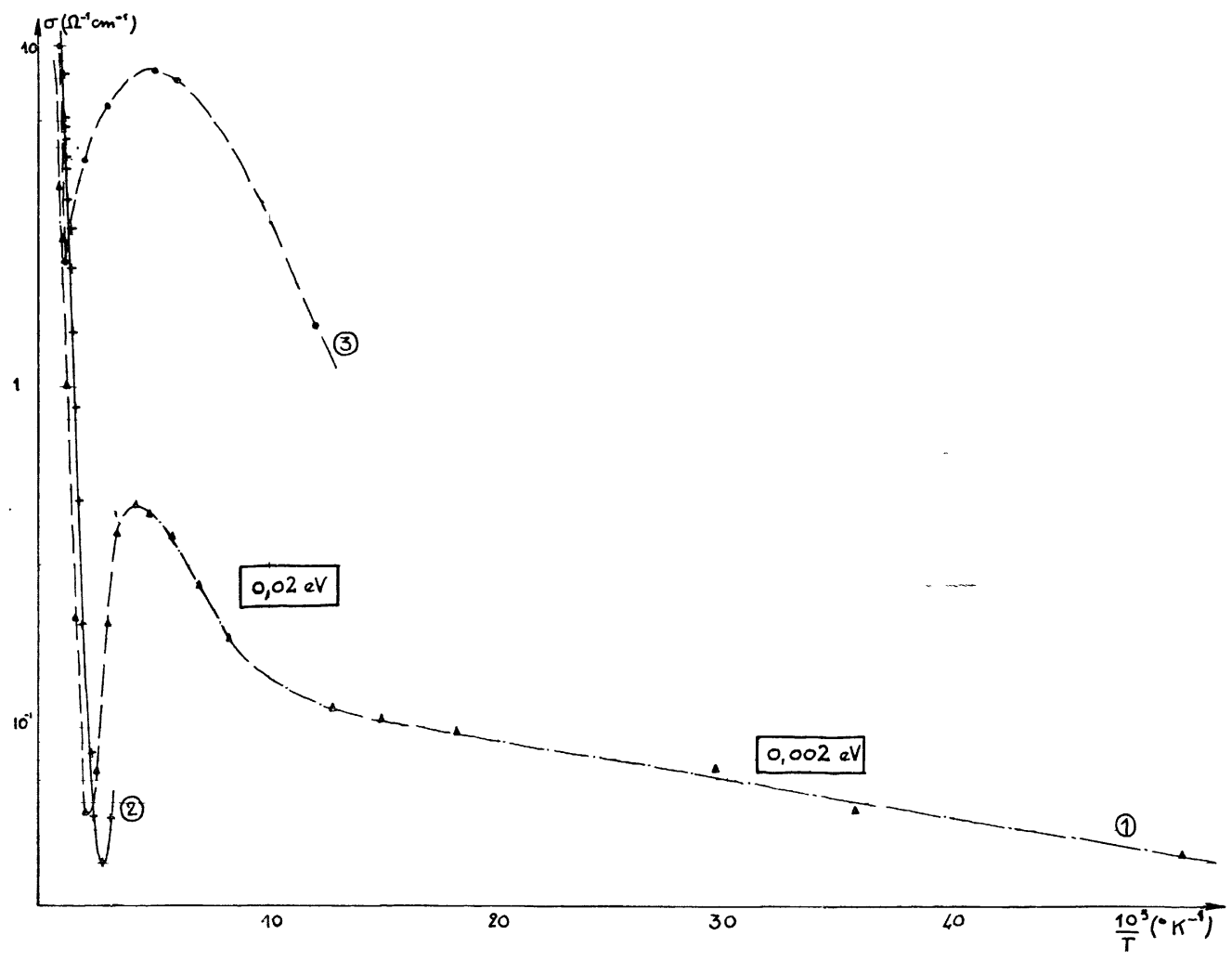

Fig. 6.

un premier niveau accepteur à $0,02 \mathrm{eV}$ se manifeste jusqu'à - $170{ }^{\circ} \mathrm{C}$; un second niveau accepteur à $2 \times 10^{-3} \mathrm{eV}$ apparaît ensuite entre $-170^{\circ} \mathrm{C}$ et $-250{ }^{\circ} \mathrm{C}\left({ }^{1}\right)$. Sur la figure 6 , nous avons aussi porté la courbe $\sigma=f(1 / T)$ obtenue par H. Welker [1] sur un échantillon massif de type $p$; la simi litude des courbes obtenues pour le matériau massif et pour les couches minces est frappante.

Il semble que le niveau donneur à $0,30 \mathrm{eV}$ soit dû à la présence d'une phase antimoine mise en évidence par l'étude aux rayons $X$ et prévue par les diagrammes de phases, ce qui concorde avec les résultats de F. Kover [12], [18].

D'autre part, un niveau accepteur à $0,02 \mathrm{eV}$ a été trouvé par Allred et al. [19]. Par contre le niveau à $2 \times 10^{-3} \mathrm{eV}$ ne semble pas avoir été remarqué par d'autres auteurs.

c) Conclusion. - Après recuit à $650^{\circ} \mathrm{C}$, les pro-

(1) Ces mesures à basse température ont été faites au Laboratoire du Professeur Pesteil, que nous tenons à remercier très vivement. priétés semiconductrices des couches minces d'AlSb que nous arons obtenues s'apparentent aux résultats trouvés pour le matériau massif.

Toutes ces couches sont de type $p$, les conductibilités variant entre $5 \times 10^{-2}$ et $5 \times 10^{-1} \Omega^{-1} \cdot \mathrm{cm}^{-1}$ à la température ambiante.

IV. Propriétés optiques et photoélectriques. Les courbes de transmission dans l'infrarouge et le visible ont été tracées au spectrophotomètre à double faisceau. Parallèlement nous avons effectué des mesures du facteur de réflexion.

Pour $T_{\mathbf{R}}<650^{\circ} \mathrm{C}$, la largeur de bande interdite optique $\Delta E_{0}$ obtenue par application du critère de Moss est de l'ordre de $1,90 \mathrm{eV}$. Pour les couches stabilisées $\left(T_{\mathrm{R}}>650^{\circ} \mathrm{C}\right)$, une transition indirecte nécessitant une énergie d'activation $\Delta E_{0}^{\prime} \simeq 1,50 \mathrm{eV}$ se manifeste, masquant quelque peu la transition à $1,90 \mathrm{eV}$, toujours présente. Les courbes de sensibilité spectrale de la photoconduction, dont l'allure générale est donnée par la courbe de la figure 7, confirment cette structure énergétique. Ces résul- 


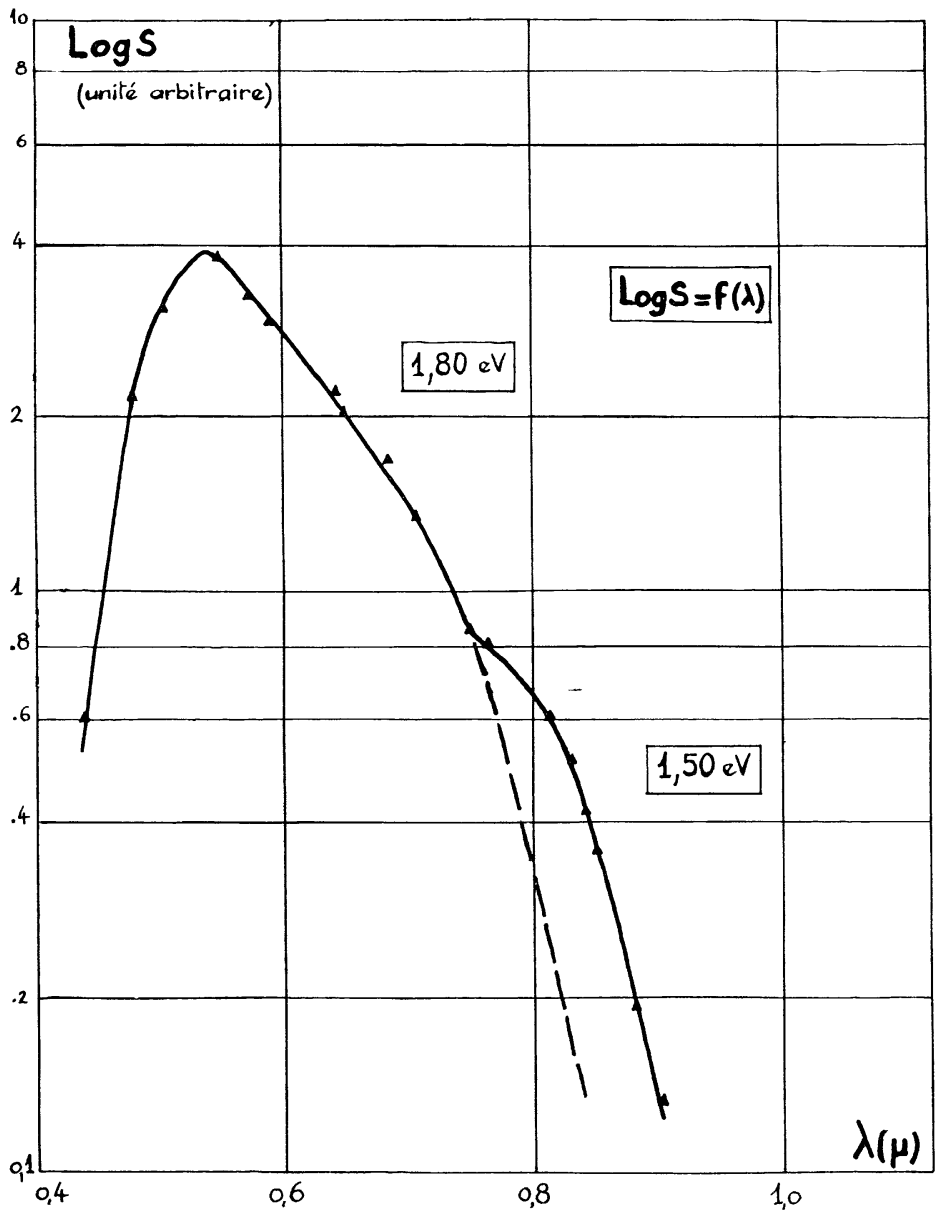

FIG. 7.

tats sont en bon accord avec le modèle proposé par Turner [20] pour la structure de bandes d'AlSb. En effet l'énergie d'activation de $1,90 \mathrm{eV}$ serait associée à la transition directe pour $\mathbf{k}=0$, tandis que $\Delta E_{0}^{\prime}=1,60 \mathrm{eV}$ correspondrait à une transition assistée entre le sommet de la bande de valence et le minimum le plus bas de la bande de conduction (ces valeurs étant prises à 0 o K).

Conclusion. - Nous avons donc pu préparer, par évaporation thermique simultanée et décalée des éléments constituants, des couches minces d'AlSb dont les propriétés semiconductrices s'apparentent à celles du matériau massif. Les couches obtenues par évaporation décalée paraissent sensiblement meilleures.

Actuellement nous nous proposons d'obtenir des couches minces orientées de ce composé sur des supports monocristallins, de poursuivre l'étude de leurs propriétés électriques et optiques, et enfin d'aborder celle des couches ternaires AlSbIn et AlSbGa.

\section{BIBLIOGRAPHIE}

[1] Welker (H.), Z. Naturforsch., 1953, 8a, 248.

[2] Justr (E.) et Lautz (G.), Abhandl. Braunschsveig. Wiss. Ges., 1953, 5, 36.

[3] Cunnel (F. A.), Edmond (J. T.) et Richards (J. L.), Proc. Phys. Soc., 1954, 87 B, 848.

[4] Blunt (R. F.), Hosler (W. R.) et Frederikse (H. P. R.), Phys. Rev., 1954, 96, 576.

[5] Briggs (H. B.), Cummings (R. F.), Hrostowski (H. R.) et Tanenbaum (M.), Phys. Res., 1954, 93, 912 .
[6] Blunt (R. F.), Frederikse (H. P. R.), Becker (J. H.) et Hosler (W. R.), Phys. Rev., 1954, 96, 578.

[7] Werker (H.), Physica, 1954, 20, 893.

[8] Hrostowski (H. J.) et Tanenbaum (J. M.), Physica, 1954, 20, 1065.

[9] Breckenridge (R. G.) et al., Physica, 1954, 20, 1073.

[10] Willardson (R. K.), Beer (A. G.) et Middleton (A. E.), J. Electrochem. Soc., 1954, 101, 354. 
[11] Sasaki (W.), Sakamoto (N.) et Kuno (M.), J. Phys. Soc. Japan, 1954, 9, 650.

[12] Kover (F.), C. R. Acad. Sc., 1956, 243, 648.

[13] Nasledov (D. N.) et Slobotchikov (S. V.), Zhr. Tekh. Fiz., 1958, 28, 4.

[14] Allred (W. P.), Paris (B.) et Genser (M.), J. Electrochem. Soc., 1958, 105, 93.

[15] Herzog (A.), Haberecht (R. R.) et Middleton (A. E.), J. Electrochem. Soc., 1958, 105, 533.

[16] Reid (F. J.) et Willardson (R. K.), J. Electronics and Control, 1958, 5, 54.

[17] Nasledov (D. N.) et Slobotchikov (S. V.), Fiz. To. Tela, 1959, 1, 748.

[18] Kover (F.), Solid St. Physics in Electronics and Telecom., Academic Press, N. Y., 1960, vol. 2, 768.

[19] Allred (W. P.), Mefferd (W. L.) et Willardson (R. K.), J. Electrochem. Soc., 1960, 107, 117.

[20] Turner (W. J.) et Reese (W. E.), Phys. Rev., 1960, 117, 1003.
[21] Hannay (B. N.), Semiconductors, Reinhold Publ. Corp., N. Y., 1960, p. 127.

[22] Michel (P.), C. R. Acad. Sc., 1956, 243, 2063.

[23] Presnov (V. A.) et Synorov (V. F.), Zhur. Tekh. Fiz., 1957, 27, 123.

[24] Sorokin (G. P.), Izo. Vysshykh. Uchebn. Zarednii, Khim. i Khim. Technol., 1962, 5, 407.

[25] Johnson (J. E.), J. Appl.Phys., 1965, 36, 10, 3193.

[26] LAude (L.), Bull. COMPLES, 1965, no 9, 83.

[27] Welker (H.) et Weiss (H.), Sol. St. Physics, vol. 3, p. 1.

[28] Guertler (W.) et Bergman (A.), Z. Metallkunde, $1933,25,81$.

[29] Liv (T. S.) et Peretti (E. A.), Trans. Am. Soc. Metals, 1952, 44, 539 .

[30] Moss (T. S.), Photoconductivity in the elements, Butterworth Sc. Publ., London, 1952. 